\title{
Natural Variation of Flowering Time and Vernalization Responsiveness in Brachypodium distachyon
}

\author{
Christopher J. Schwartz • Mark R. Doyle • \\ Antonio J. Manzaneda • Pedro J. Rey • \\ Thomas Mitchell-Olds • Richard M. Amasino
}

Published online: 7 February 2010

(C) Springer Science+Business Media, LLC. 2010

\begin{abstract}
Dedicated bioenergy crops require certain characteristics to be economically viable and environmentally sustainable. Perennial grasses, which can provide large amounts of biomass over multiple years, are one option being investigated to grow on marginal agricultural land. Recently, a grass species (Brachypodium distachyon) has been developed as a model to better understand grass physiology and ecology. Here, we report on the flowering time variability of natural Brachypodium accessions in response to temperature and light cues. Changes in both environmental parameters greatly influence when a given accession will flower, and natural Brachypodium accessions broadly group into winter and spring annuals. Similar to what has been discovered in wheat and barley, we find that a portion of the phenotypic variation is associated with changes in expression of orthologs of $V R N$ genes, and thus, $V R N$ genes are a possible target for modifying flowering time in grass family bioenergy crops.
\end{abstract}

Electronic supplementary material The online version of this article (doi:10.1007/s12155-009-9069-3) contains supplementary material, which is available to authorized users.

C. J. Schwartz $\cdot$ M. R. Doyle $\cdot$ R. M. Amasino $(\bowtie)$

Great Lakes Bioenergy Research Center,

Department of Biochemistry, University of Wisconsin Madison, Madison, WI 53706, USA

e-mail: amasino@biochem.wisc.edu

\section{A. J. Manzaneda $\cdot$ T. Mitchell-Olds}

Institute for Genomic Sciences and Policy,

Department of Biology, Duke University,

Durham, NC 27708, USA

P. J. Rey

Departamento de Biologia Animal, Vegetal y Ecologia,

Universidad de Jaén,

Jaén 23071, Spain
Keywords Biomass $\cdot$ Bioenergy $\cdot$ Brachypodium .

Flowering time $\cdot$ Vernalization

\section{Introduction}

Biomass yield is an important component to consider in any program designed to derive energy from plant material. Plant size and architecture are important biomass yield parameters, and these parameters are often quite variable within a given species. Intraspecies variation in biomass yield can be a product of many factors. One such factor is the timing of the transition from vegetative to reproductive growth. The switch to flowering causes a diversion of resources from the continual production of photosynthetic material (leaves) to the terminal production of reproductive tissue (flowers, seeds, and fruit). Thus, one method to increase biomass yields is to extend the vegetative phase by delaying the transition to flowering.

The initiation of flowering during particular seasons can greatly enhance the reproductive success of different plant species. Plants have thus evolved mechanisms to sense seasonal environmental cues that serve as a signal to initiate the conversion from vegetative to reproductive growth. For example, many species flower in response to changes in photoperiod, the relative length of day and night (for review, see $[1,2])$. Others require exposure to the prolonged cold of winter to promote flowering through a process known as vernalization (for review, see [3]).

In the model dicot Arabidopsis thaliana (hereafter referred to as Arabidopsis), the genetic basis of flowering time has been studied extensively, and a plethora of genes have been implicated in the control of flowering time, but the majority of flowering time variation in Arabidopsis is attributable to just a few loci. Molecular 
studies of flowering-time control in cereals such as wheat and barley demonstrate that there are some similarities, but also important differences, between monocots and dicots [4].

Genetic analysis of flowering in wheat and barley has led to the identification of three VERNALIZATION (VRN) loci that influence the flowering response to cold for these cool season grasses. VRN1 is a MADS-box transcription factor similar to the Arabidopsis genes APETALA1 and FRUITFUL [5]. Expression of VRN1 is induced by cold, and VRN1 represses the expression of VRN2. VRN2 encodes a small CCT-domain protein that acts to repress flowering. Allelic variation at $V R N 2$ is often responsible for the difference in vernalization requirement in winter versus spring varieties [6]. VRN2 acts to repress $V R N 3$, a homolog of the Arabidopsis FLOWERING LOCUS T (FT) gene, a strong promoter of flowering [7]. Photoperiod also strongly influences flowering time in the grasses, as demonstrated by studies in rice (Oryza sativa L.). In rice, the Arabidopsis $F T$ homolog (Heading date $3 a$ ) is induced under permissive photoperiods and promotes flowering [8]. To date, FT appears to be universal floral promoter. In all plants where flowering-time genes have been investigated, orthologs of FT promote flowering [1].

Perennial grasses, such as Miscanthus (Miscanthus giganteus) and switchgrass (Panicum virgatum), are promising bioenergy crops because of the potential for high biomass yields with low inputs, traits which are key for economic feasibility and environmental sustainability. Switchgrass is a prairie grass native to and widespread in North America which is capable of growth on marginal agricultural lands (http://bioenergy.ornl.gov/papers/misc/ switgrs.html). Determining which loci and the combinations of those loci that control the genetic basis of flowering time in perennial grasses could greatly facilitate the development of cultivars that produce maximal biomass for a given location. However, many of the perennial grasses being considered for dedicated bioenergy crops are difficult models for gene identification due to polyploidy, obligate out crossing, long life cycle, and large size.

In 2008, the Department of Energy Joint Genome Institute sequenced the genome of the grass Brachypodium distachyon (hereafter referred to as Brachypodium), with the objective of making this species a genetic model for grasses. Brachypodium has a number of physical attributes that make it suitable for a model organism, such as a small genome, diploid strains, short life cycle, transformability, and small size [9-11]. In addition to a complete genome sequence, additional genetic and molecular resources include an expressed sequence tag and bacterial artificial chromosome libraries, microarrays, collections of natural strains, and the development of mutant populations (http:// www.brachypodium.org/). Brachypodium has also been shown to be syntenic with other grass genomes making it applicable for comparative genomics [12-14].

Brachypodium accessions are highly variable for flowering time and biomass production (Fig. 1), but a vernalization treatment reduces this variability by reducing the time needed for winter-annual accessions to flower [10, 11, 15, 16]. Information concerning the loci responsible for flowering-time differences in natural Brachypodium accessions is limited. Using the rice $H d 3 a$ ( $F T$ ortholog) sequence as a probe, it was demonstrated that in Brachypodium, FT exists as a single copy gene [13]. Transformation of different Brachypodium accessions with the floral repressor Terminal Flower 1 from Arabidopsis or perennial ryegrass (Lolium perenne L.) showed that both genes are functional in Brachypodium and cause a delay in flowering time $[15$, 17-19].

In this study, we investigate the flowering behavior of natural accessions of Brachypodium in response to cold and photoperiod treatments. We report substantial variation in flowering time among Brachypodium accessions, which indicates potential for using natural variation to uncover genes that function in the regulation of flowering in grasses. We also present an initial characterization of putative VRN orthologs in Brachypodium.

\section{Materials and Methods}

\section{Plant Materials}

Brachypodium accessions used in this study are listed in Table 1. Seeds of Bd1-1, Bd2-3, Bd3-1, Bd18-1, and Bd21 were kindly provided by David Garvin (USDA-ARS, Plant Science Research Unit and Department of Agronomy and Plant Genetics, University of Minnesota, St. Paul, MN, USA). Seeds of BdCzPP-2, BdCzPP-3, DSNSCA-6, and RON-2 were collected in Spain in the summer of 2007 by A. Manzaneda. Seeds from diploid accessions collected in Turkey were kindly provided by Hikmet Budak (Sabanci University, Istanbul, Turkey) and John Vogel (USDA-ARS, Western Regional Research Center, Albany, CA, USA [20]).

Plant Materials and Growth Conditions

Seeds of Bd1-1, Bd2-3, Bd3-1, Bd18-1, Bd21, BdCzPP-2, BdCzPP-3, DSNSCA-6, RON-2, and the Turkish accessions were imbibed overnight between wet paper towels in the dark at $4^{\circ} \mathrm{C}$. Seeds were planted in Metro-Mix 360 (SUN GRO Horticulture) and grown for 4 weeks at $20^{\circ} \mathrm{C}$ with $20 \mathrm{~h}$ of light per day at a fluence rate of 60 $70 \mu \mathrm{mol} \mathrm{m} \mathrm{s}^{-1}$. Seedlings were then moved to one of four conditions: (1) no vernalization, (2) 2 weeks of vernaliza- 


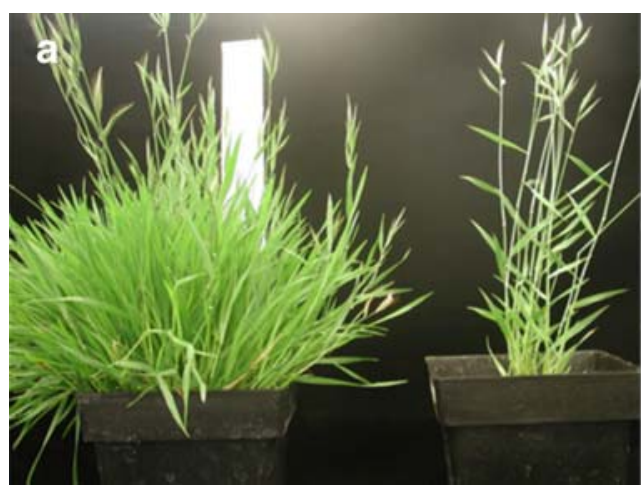

Fig. 1 Variability in biomass accumulation among Brachypodium accessions. a Single plant from two different accessions, which flowered on the same date. The accession on the left (Bd1-1) required 5 months to flower and had more biomass compared to the accession

Table 1 Accessions used in this study

\begin{tabular}{|c|c|c|c|}
\hline Origin & Bd inbred lines & Seed origin & Ploidy \\
\hline Turkey & Bd1-1 & Garvin [10] & Diploid [34] \\
\hline Iraq & $\mathrm{Bd} 2-3$ & Garvin $[10]$ & Diploid [34] \\
\hline Iraq & $\mathrm{Bd} 3-1$ & Garvin $[10]$ & Diploid [34] \\
\hline Turkey & Bd18-1 & Garvin $[10]$ & Diploid [34] \\
\hline Iraq & $\mathrm{Bd} 21$ & Garvin $[10]$ & Diploid [34] \\
\hline Iraq & $\mathrm{Bd} 21-3$ & Vogel [35] & Diploid [34] \\
\hline Spain & $\mathrm{BdCzPP}-2$ & TMO & Polyploid \\
\hline Spain & $\mathrm{BdCzPP}-3$ & TMO & Polyploid \\
\hline Spain & RON-2 & TMO & Diploid \\
\hline Spain & DSNSCA-6 & TMO & Diploid \\
\hline Turkey & $\operatorname{BdTR} 1(\mathrm{a}, \mathrm{b}, \mathrm{c}, \mathrm{d}$, and e $)$ & Budak [20] & Diploid [20] \\
\hline Turkey & $\operatorname{BdTR} 2(\mathrm{a}, \mathrm{c}, \mathrm{d}$, and e) & Budak [20] & Diploid [20] \\
\hline Turkey & $\operatorname{BdTR} 3(a, b, c, d$, and e) & Budak [20] & Diploid [20] \\
\hline Turkey & $\operatorname{BdTR} 5(a, b, c$, and e) & Budak [20] & Diploid [20] \\
\hline Turkey & BdTR7 (a and b) & Budak [20] & Diploid [20] \\
\hline Turkey & $\operatorname{BdTR} 8(a, c, f, i$, and $m)$ & Budak [20] & Diploid [20] \\
\hline Turkey & $\operatorname{BdTR} 9(a, b, c$, and e) & Budak [20] & Diploid [20] \\
\hline Turkey & $\operatorname{BdTR} 10(a, b, c, d$, and e) & Budak [20] & Diploid [20] \\
\hline Turkey & $\operatorname{BdTR} 11(\mathrm{a}, \mathrm{b}, \mathrm{c}, \mathrm{d}$, and e $)$ & Budak [20] & Diploid [20] \\
\hline Turkey & $\operatorname{BdTR} 12(a, b$, and $c)$ & Budak [20] & Diploid [20] \\
\hline Turkey & $\operatorname{BdTR} 13$ (a, b, c, d, and e) & Budak [20] & Diploid [20] \\
\hline Turkey & Adi $(1,2,3,4$, and 5$)$ & Vogel [32] & Diploid [32] \\
\hline Turkey & Bis-1 & Vogel [32] & Diploid [32] \\
\hline Turkey & $\operatorname{Gaz}(1,2,3,4$, and 5$)$ & Vogel [32] & Diploid [32] \\
\hline Turkey & $\operatorname{Kah}(1,2,3,4$, and 5) & Vogel [32] & Diploid [32] \\
\hline Turkey & $\operatorname{Koz}(1,2,3,4$, and 5$)$ & Vogel [32] & Diploid [32] \\
\hline Turkey & Tek $(1,2,3,4$, and 5) & Vogel [32] & Diploid [32] \\
\hline
\end{tabular}

For the Turkish accessions from Vogel and Budak, three to five isolates from each location were pooled for the phenotypic analysis, due to limited seed supply

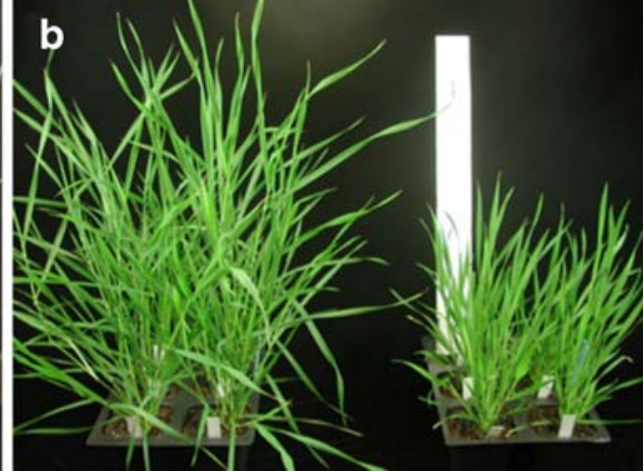

on the right (Bd21), which required only 2 months to flower. b Two different accessions at 2 months, with the accession on the left (BdCzPP-2) growing faster and producing more biomass in a given time compared to the accession on the right $(\mathrm{Bd} 21)$

tion (not tested with Turkish accessions), (3) 4 weeks of vernalization, and (4) 6 weeks of vernalization. Seedlings were vernalized in a cold room at $4^{\circ} \mathrm{C}$ in short days (SD; $8: 16 \mathrm{~h} \mathrm{~L} / \mathrm{D})$, with the exception of the Turkish accessions for which seedlings were vernalized under constant light conditions. Plants were subsequently placed into a growth chamber containing cool-white fluorescent bulbs (GE EcoLux F32T8) at $20^{\circ} \mathrm{C}$ in long days (LD; 20:4 h L/D) and flowering time scored. For the Turkish accession, phenotypic data represents five isolates, for example the phenotypic value for BdTR1 was determined by scoring the flowering time from 3 plants each of BrTR1a, BdTR1b, BdTR1c, BdTR1d, and BdTR1e. In photoperiod shift experiments, long days were $20: 4 \mathrm{~h} \mathrm{~L} / \mathrm{D}$, and short days were $8: 16 \mathrm{~h} \mathrm{~L} / \mathrm{D}$.

For studies of seed vernalization, seeds $(n=8)$ from accession Bd1-1 were surface sterilized by rinsing $2 \times$ in $70 \% \mathrm{EtOH}$, followed by two rinses in $\mathrm{dH} 2 \mathrm{O}$. Seeds were soaked in a flask with gentle swirling for $1 \mathrm{~h}$ prior to plating. Seeds were plated on agar-solidified medium as described previously [21] and placed at $4^{\circ} \mathrm{C}$ for $24 \mathrm{~h}$. Seed were moved to one of four conditions: (1) no vernalization, (2) 2 weeks of vernalization, (3) 4 weeks of vernalization, and (4) 6 weeks of vernalization. Seeds were vernalized at $4^{\circ} \mathrm{C}$ with in short days $(8: 16 \mathrm{~h} \mathrm{~L} / \mathrm{D})$. After the treatment, seeds had no visible root emergence and were sowed in MM360 and placed at $20^{\circ} \mathrm{C}$ in long days $(20: 4 \mathrm{~h} \mathrm{~L} / \mathrm{D})$.

To test the effects of far-red enrichment on plant architecture and flowering time, 4-week-old plants were placed in long days (20:4 h L/D) supplemented with far-red LED bulbs. The ratio of red/far-red under these conditions is 0.15 compared to the unenriched conditions which have a ratio of 5 .

\section{Genotyping and Expression Analysis}

Oligos were designed for polymerase chain reaction (PCR) that include the polymorphic region of intron1 of 
Bradi3g10010. Once amplified, PCR products were digested with $M s e I$ and run out on a $4 \%$ agarose gel. Digested DNA resulted in unique banding patterns for all four Bradi3g10010 classes.

RNA was prepared from two separate pools of 7-day-old seedlings $(n=3)$, harvested at 4 PM (corresponding to the time of peak VRN3/FT expression) using the Nucleospin RNAII kit (Clontech) according to the manufacturer's instructions. cDNA synthesis was carried out using MMLV reverse transcriptase (Promega) according to the manufacturer's instructions. Quantitative reverse transcription PCR (qRT-PCR) was performed with the 7000 Real Time PCR System (Applied Biosystems) using the DyNAmo $^{\mathrm{TM}}$ Flash SYBR ${ }^{\circledR}$ Green qPCR Kit (Finnzymes). The PCR parameters were one cycle of 15 min at $95^{\circ} \mathrm{C}, 40$ cycles of $15 \mathrm{~s}$ at $95^{\circ} \mathrm{C}, 20 \mathrm{~s}$ at $58^{\circ} \mathrm{C}$, and $45 \mathrm{~s}$ at $72^{\circ} \mathrm{C}$. The constitutively expressed putative Brachypodium homolog of UBIQUITIN-CONJUGATING ENZYME 18 (UBC18 Bradi4g00660) was used as a reference for gene expression as described [22]. PCR primer sequences are listed in Table 2. All results presented are an average of two biological replicates.

\section{Statistical Analysis}

ANOVAs and $p$ test were performed using Microsoft Excel. Least significant difference was calculated for each condition, using a weighted mean to correct for uneven sample size. Standard deviations were calculated using Microsoft Excel.

Table 2 Sequences of oligos used in this study

$$
\begin{aligned}
& \text { Genotyping Oligos } \\
& \text { Bradi3g10010 intron 1 F } \\
& \text { Bradi3g10010 intron 1 R }
\end{aligned}
$$

\begin{tabular}{|c|c|}
\hline $\begin{array}{l}\text { Homologous gene in } \\
\text { cereals }\end{array}$ & Gene ID \\
\hline \multirow[t]{2}{*}{ VRN1 } & Bradi1g08340 F \\
\hline & Bradi1g08340 R \\
\hline \multirow[t]{2}{*}{ VRN1 } & Bradi1g59250 F \\
\hline & Bradi1g59250 R \\
\hline \multirow[t]{2}{*}{ VRN2 } & Bradi3g10010 F \\
\hline & Bradi3g10010 R \\
\hline \multirow[t]{2}{*}{ VRN3 } & Bradi1g48830 F \\
\hline & Bradi1g48830 R \\
\hline
\end{tabular}

Quantitative Real-Time PCR Oligos

\section{Results and Discussion}

The Vernalization Response in Brachypodium Accessions

Flowering time was analyzed for 24 natural accessions of $B$. distachyon in two independent experiments (Table 1). Nine accessions isolated from various geographical locations were assayed for flowering time following $0,2,4$, or 6 weeks of vernalization (Fig. 2), and 15 accessions collected from Turkey were similarly assayed after 0,4 , or 6 weeks of vernalization (Fig. 3). For these two independent experiments, young plants (3 weeks old) were vernalized. In both experiments, we observed variation between strains for flowering time in both vernalized and nonvernalized samples. However, vernalization led to a decrease in variation within and between accessions, and a decrease in the numbers of days required to flower for most accessions (Figs. 2 and 3). Some of the Turkish strains displayed variation within the line (data not shown), indicating that there may be flowering-time loci segregating in these lines (BdTR12 and BdTR9). These lines were not included in our analysis. In contrast to the other accessions, the Turkish accession contains multiple isolates and individual isolates are not genetically homogeneous [20, 32]. However, even for the genetically heterogenous Turkish accessions, an ANOVA of the data shows that the vast majority of the variability observed was between accessions. Three accessions (Bd21, Bd3-1, DSNSCA-6) behaved as spring annuals, in which the accession would flower without vernalization and a

\section{AATTTGCTTGCTATATGCCTGT}

GGAGGATAGGAGTACGGACAGTAA

Oligo Sequence
GTCGCGCTCATCATCTTCTC
TGCATAGGAGTAGCGCTCATAG
CAAGGGCAAGCTCTACGAGT
AAGAGCCTTTTCAGCATACGA
GTTGAACCGGCCACTCAT
CATGGTGTCGCCAAATGT
GCAATGAGATGAGGACCTTCT
TGGAATATCTGTCACTAACCAGTGT

Genotyping oligos were used to assay deletion polymorphisms in the first intron of Bradi3g10010. qRT-PCR oligos were used to quantify gene expression (Fig. 6) 


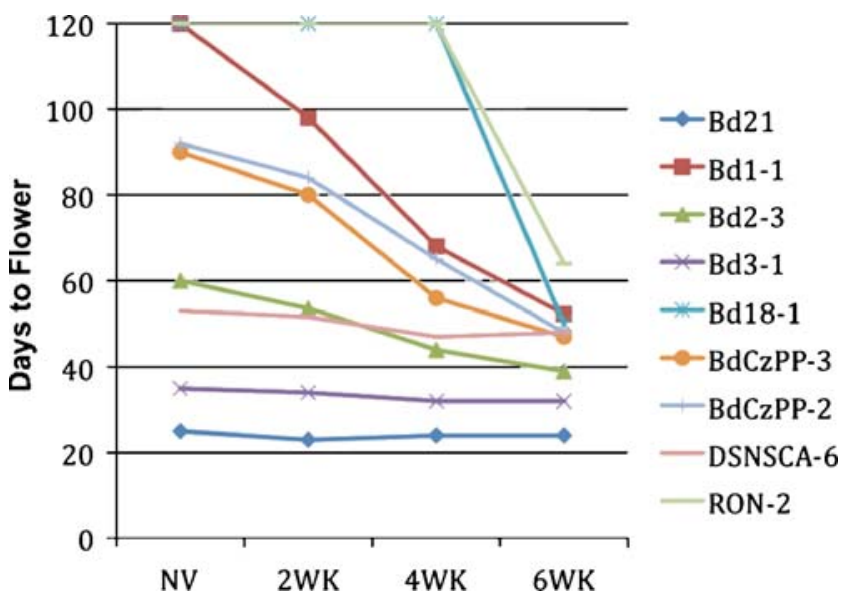

Fig. 2 Flowering time and vernalization responsiveness for nine Brachypodium accessions. Flowering time was determined after 0, 2, 4 , or 6 weeks of a vernalization treatment as seedlings. Each time point consisted of three to six plants, and the experiment was terminated 120 days after the vernalization treatments. Accessions $\mathrm{Bd} 21$ and $\mathrm{Bd} 21-3$ exhibited identical profiles (data not shown); therefore, only $\mathrm{Bd} 21$ is included. Data points at 120 days represent plants that did not flower during the course of the experiment. Least significant differences for the four treatments are $(\alpha=0.05) ; N V=$ $6.055,2 W K=4.986,4 W K=4.284$, and $6 W K=3.558$

vernalization treatment did not greatly accelerate the flowering time (Fig. 2). Three accessions behaved as winter annuals (Bd1-1, Bd18-1, and RON-2), and these accessions are quite late flowering (or in our conditions do not flower) without a vernalization treatment, and vernalization of these accessions results in a large decrease in flowering time (Fig. 2). However, there is substantial variation within the spring and winter-annual classes, indicating that flowering might be controlled by multiple loci and/or alleles. Two polyploid accessions (BdCzPP-2 and $\mathrm{BdCzPP}-3$ ) were intermediate for flowering time relative to the spring and winter annuals, and for these accessions, a vernalization treatment reduced the time to flower, suggesting that their growth habits are more similar to the winter-annual accessions.

The ability of certain species to undergo vernalization as an imbibed seed is common in cereals, whereas in other species, vernalization is only possible in plants that have reached a certain stage of maturity [23, 24]. Seed vernalization of wheat was used extensively in the last century to essentially convert winter varieties into hardy spring varieties, ensuring a stable food supply in regions of the world in which only winter varieties could survive spring plantings [25]. Because many cereals can be vernalized as seed, we wanted to determine if the same holds true for Brachypodium. We evaluated the vernalization response in imbibed seeds of a single accession (Bd1-1) in order to compare the response of vernalization to that seen in young plants (Fig. 4). Seed vernalization of Bd1-1 had the same promoting effect as in young plants demonstrating that imbibed seeds are also capable of responding to a vernalization treatment. Specifically, 2 weeks of cold treatment causes a slight decrease in flowering time, but the within variability is relatively high, similar to that seen in the nonvernalized plants. However, after 4 weeks of vernalization, the flowering time was reduced to less than half the time it took the nonvernalized plants to flower, and the variability is also greatly reduced. Six weeks of vernalization caused even earlier flowering, but the difference between 4 and 6 weeks $(p=0.053)$ was much less compared to the flowering time difference between 2 and 4 weeks $(p=0.003)$, indicating that the response in this accession is nearing saturation by 6 weeks.
Fig. 3 Flowering time and vernalization responsiveness for 15 Turkish Brachypodium accessions. Flowering time was determined for the accessions following 0,4 , or 6 weeks of vernalization as seedlings. Each time point consisted of six to 15 plants, and the experiment was terminated 120 days following the vernalization treatment. $\mathrm{Col}$ umns without error bars (standard deviation) contained at least one plant that did not flower during the experiment

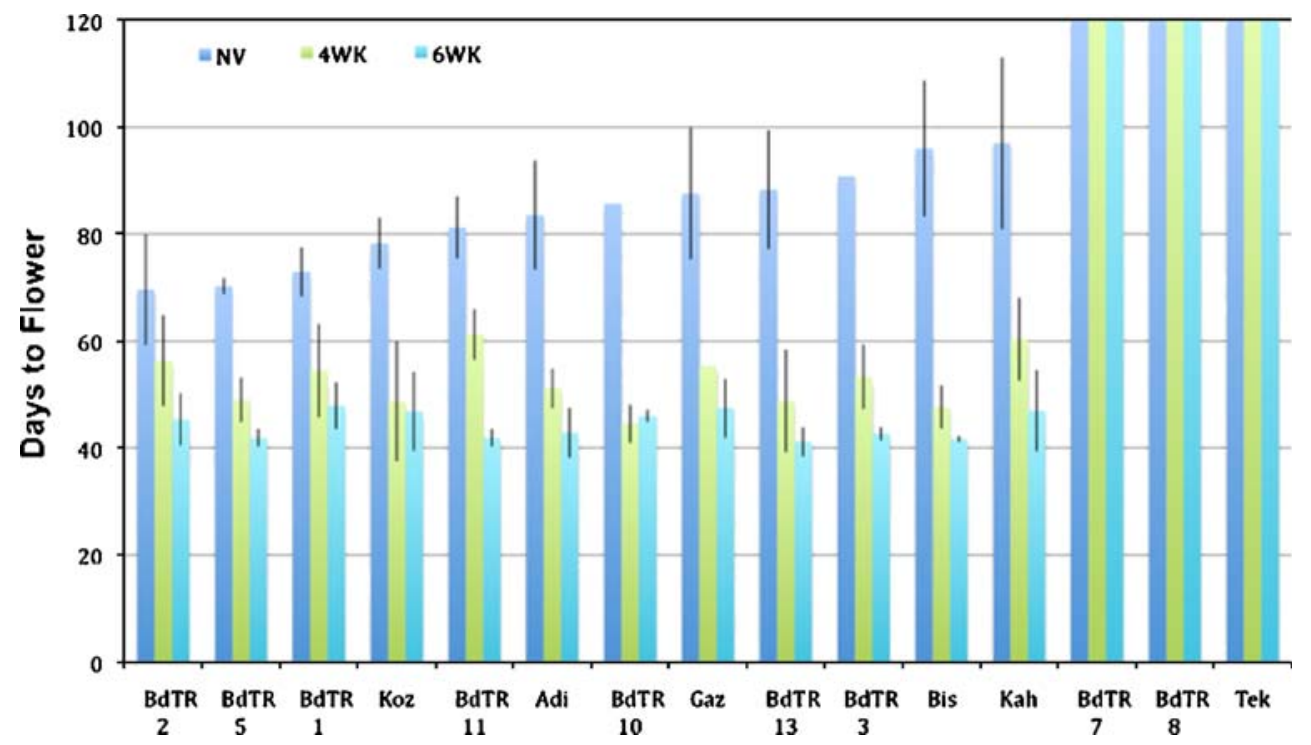




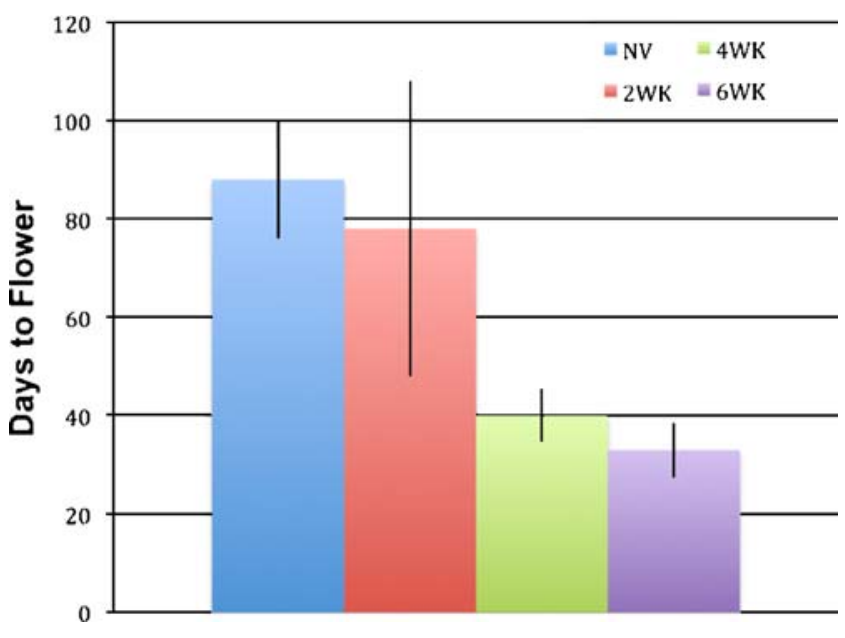

Fig. 4 Flowering time of accession Bd1-1 vernalized as seeds. Flowering time was determined for accession Bd1-1 after 0, 2, 4, or 6 weeks of vernalization as seed (see "Materials and methods" section). Each column represents eight plants, and error bars are the standard deviation

Response to Photoperiod and Light Quality

In both wheat and barley, exposure of certain winter accessions to SD can hasten the transition to flowering in the absence of cold, i.e., SD can serve as a substitute for vernalization $[26,27]$. Thus, we wanted to determine the effect of a SD treatment on flowering in Brachypodium accessions. Accordingly, four accessions with varying flowering times were germinated under long days. After 2 weeks of growth in LD, plants were given one of three treatments: (1) maintained in LD, (2) transferred to SD for

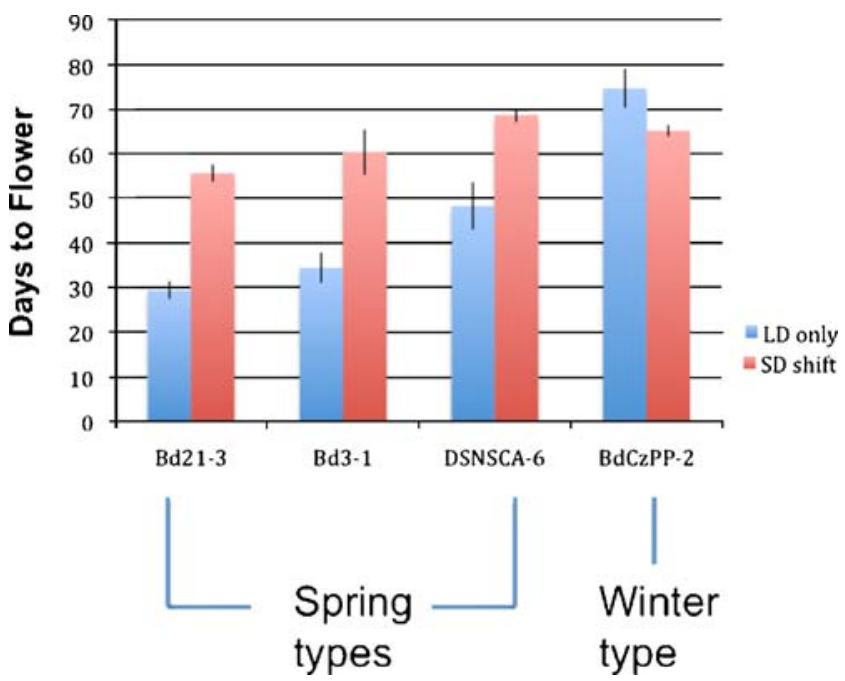

Fig. 5 A temporary photoperiod shift to SD accelerates flowering in winter-annual Brachypodium accessions. Plants were grown in LD for 2 weeks and were either (1) maintained in LD or (2) shifted to SD for 4 weeks and then returned to LD. The SD shift only accelerated flowering in the winter-annual accession. Days to flower represents the time for flowering to occur from when the seeds germinated
4 weeks and then returned to LD, or (3) transferred to and maintained in SD. All of the spring-annual accessions showed a similar delay in flowering when subjected to a SD shift; however, accession BdCzPP-2, which has a robust vernalization response, flowered earlier in response to the temporary SD shift (Fig. 5), whereas none of the accessions flowered when maintained in SD. Therefore, there appears to be variation in the efficacy of a temporary SD shift to promote flowering in Brachypodium, and we identified one accession in which SD substituted, at least partially, for vernalization. Future experiments will address the effects of SD on additional Brachypodium accessions as well as the optimal photoperiod length of the SD and the optimal length of time in SD required for the promotion of flowering.

In Arabidopsis, nearby vegetation (shade) causes a number of phenotypic changes, which has been collectively called the shade avoidance syndrome. These include plant elongation and an acceleration of flowering. Six Brachypodium accessions were tested under enriched far-red conditions, which mimic shade, and neither plant elongation nor accelerated flowering was observed (data not shown). Therefore, the specific R/FR ratio that triggers the shade avoidance syndrome in Arabidopsis does not have the same affect in Brachypodium, which is consistent with the fact that

Table 3 Genotype classes of the BdVRN2 intron (Supplemental Figure 1) in different Brachypodium accessions

\begin{tabular}{|c|c|c|c|}
\hline Class 1 & Class 2 & Class 3 & Class 4 \\
\hline $\begin{array}{l}\mathrm{Bd} 21 \\
\mathrm{Bd} 3-1 \\
\mathrm{Bd} 21-3\end{array}$ & $\begin{array}{l}\text { Bd1-1 } \\
\text { BdCzPP_3 } \\
\text { BdTR8I } \\
\text { Tek-1 } \\
\text { Tek-3 } \\
\text { Tek-5 }\end{array}$ & $\begin{array}{l}\text { Bd18-1 } \\
\text { Bd2-3 } \\
\text { BdCzPP-2 } \\
\text { DSNSCA_6 } \\
\text { RON-2 } \\
\text { BdTR1 } \\
\text { BdTR2 } \\
\text { BdTR3 } \\
\text { BdTR5 } \\
\text { BdTR9 } \\
\text { BdTR10 } \\
\text { BdTR11 } \\
\text { BdTR12 } \\
\text { BdTR13 } \\
\text { Adi } \\
\text { Bis } \\
\text { Gaz } \\
\text { Kah } \\
\text { Koz }\end{array}$ & $\begin{array}{l}\text { BdTR7 } \\
\text { BdTR8f } \\
\text { BdTR8m } \\
\text { Tek-2 } \\
\text { Tek-4 }\end{array}$ \\
\hline
\end{tabular}

Accessions in bold behave as winter annuals. Tek and BdTR8 contained multiple isolates, and each isolate was genotyped for these two accessions 
many grasses have evolved to grow at a high vegetation density.

\section{Analysis of Putative Brachypodium VRN Orthologs}

In wheat and barley, variation in the $V R N$ genes (VRN1, $V R N 2$, and $V R N 3$ ) contributes to variation in flowering time $[28,29]$. Brachypodium contains several genes with high relatedness to wheat and barley VRN genes. When comparing both the wheat and barley VRN2 and VRN3 protein sequences to the Brachypodium annotation, single genes stood out as being most similar: Bradi3g10010 (BdVRN2) and Bradilg48830 (BdVRN3). A similar approach identified multiple genes in Brachypodium which exhibit relatedness to VRN1. This is not surprising given that VRN1 is a MADS-box transcription factor, a class of genes that exists as large gene families in flowering plants $[30,31]$. In this work, we chose to characterize the two Brachypodium genes most similar to wheat/barley VRN1, Bradilg08340 (BdVRN1L1) and Bradilg59250 (BdVRN1L2).

We sequenced BdVRN2 from several accessions and identified two indel polymorphisms ( 24 and $35 \mathrm{bp}$ ) and a single nucleotide polymorphism in the first intron (Supp Figure 1). We genotyped all available accessions for $B d V R N 2$ intron 1 polymorphisms and identified four genotypic classes (Table 3; Supplemental Figure 1). The three earliest accessions (Bd21, $\mathrm{Bd} 21-3$, and $\mathrm{Bd} 3-1$ ) comprised one genotypic class (class 1). These three accessions were unique in that a vernalization treatment did not greatly reduce flowering time. Another class (class 3) included most of the accessions analyzed in this study (19 out of 27), and 16 of these were spring annuals whereas three accessions behaved as winter annuals. The two diploid winter-annual accessions in this genotypic class, Bd18-1 and RON-2, require an extended vernalization treatment to initiate flowering (Fig. 2), indicating genetically that they may comprise a separate subgroup within this class where vernalization responsiveness and flowering time may be influenced by other loci (ex. VRN1 and VRN3). The two remaining genotypic classes (classes 2 and 4) were comprised of only winter-annual accessions $(n=5)$ and are part of a Brachypodium subgroup as determined by 143 SSR markers [32], where all the accessions are winter annuals. Therefore, there appears to be a correlation between different genotypes at $B d V R N 2$ and flowering time, but it is not known if the polymorphisms affect $B d V R N 2$ function or are just a product of population structure. Future experiments to evaluate the functionality of $B d V R N 2$ alleles include expression studies and transgenic complementation.
Fig. 6 Expression of Brachypodium putative $V R N$ orthologs. Nonvernalized seedlings were assayed for $V R N$ gene expression. Accession designations are shown on the $x$-axis and are ordered according to flowering time for the nonvernalized condition, while relative BdVRN gene expression (compared to an ubiquitin control) is shown on the $y$-axis
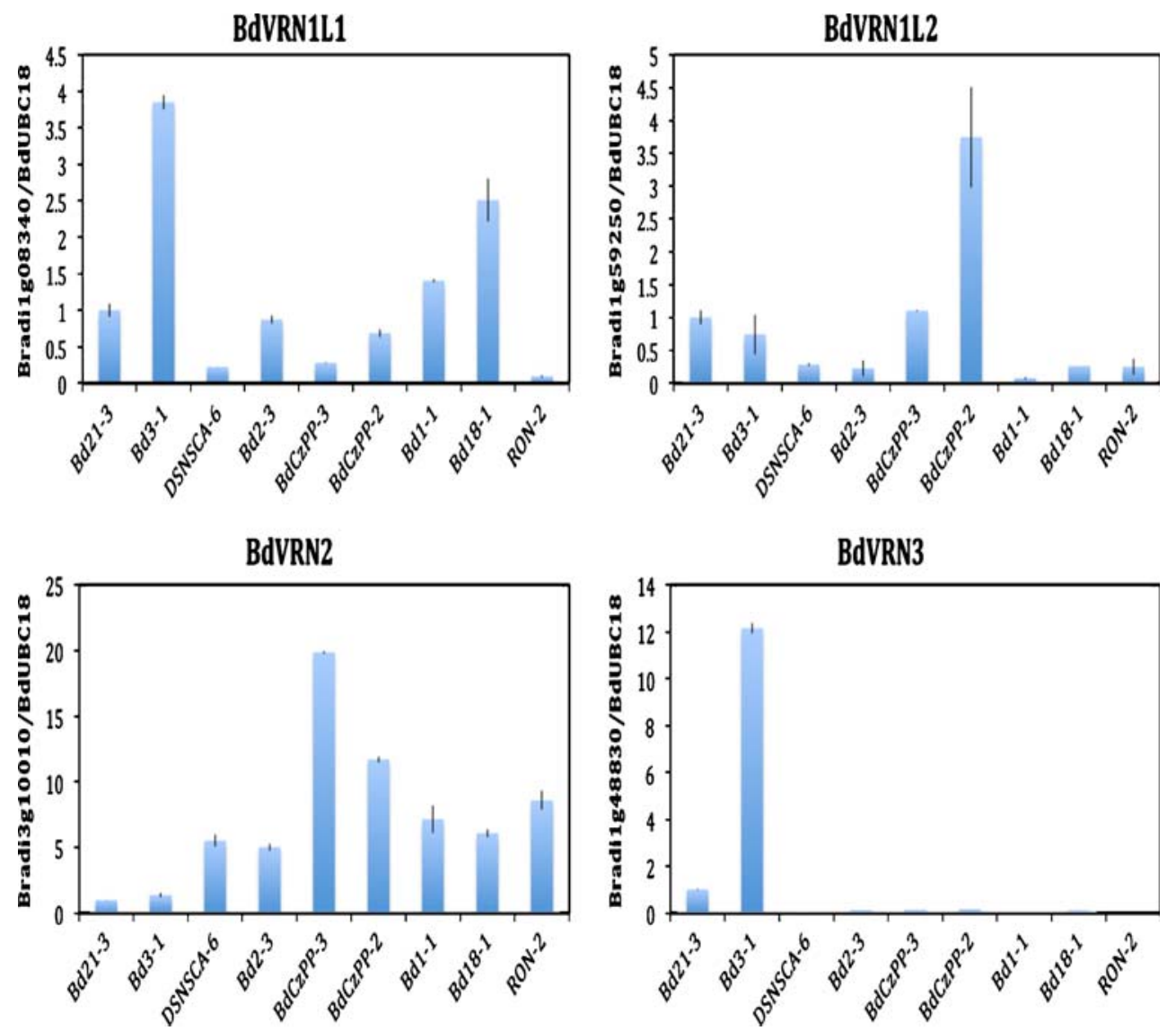
The expression level of putative Brachypodium VRN orthologs was determined by qRT-PCR. In wheat and barley, $V R N 2$ is a repressor of flowering and is expressed at lower levels in spring accessions. BdVRN2 is also expressed at lower levels in the spring-annual accessions Bd21-3 and Bd3-1 (Fig. 6). Likewise, VRN3 is a promoter of flowering and the expression of BdVRN3 is elevated in these accessions. Expression levels of Brachypodium VRN1-like genes, however, did not completely correlate with flowering time. Expression of BdVRN1L1 was elevated in the rapid-flowering $\mathrm{Bd} 3-1$ accession but was also elevated in the late flowering winter annuals $\mathrm{Bd} 1-1$ and Bd18-1. In the case of BdVRN1L2, expression levels were the highest in the late-flowering accessions BdCzPP-2 and $\mathrm{BdCzPP}-3$, but it is important to note that these are polyploid accessions, and thus, it is unclear how many copies of BdVRN1L2 exist in these backgrounds and whether these copies are functional. Among the diploid accessions, the spring annuals $\mathrm{Bd} 21-3$ and $\mathrm{Bd} 3-1$ had the highest BdVRN1L2 expression levels, whereas expression was low in the winter annuals Bd1-1 and Bd18-1. The expression data indicate that the $B d V R N 1$ genes may have different activity and roles in different accessions. Future experiments will examine changes in the expression of these genes in response to cold treatment and different photoperiod regimes.

Flowering time is an important trait to consider in the development of lignocellulosic biofuel crops as plants with delayed flowering will produce greater yields. Work done in cereals has identified several genes that regulate the transition to flowering (reviewed in [29,33]), and these loci may prove to be valuable targets for the manipulation of flowering in biofuel crops. However, domesticated grasses such as wheat and barley may have lost some variability in flowering time through the process of domestication. Being a wild grass species, Brachypodium may serve as a useful model for the identification of novel loci involved in the control of flowering. Work presented here clearly demonstrates a large degree of variation in flowering time among natural accessions of Brachypodium.

Brachypodium is a genetically tractable system to test genes involved in flowering time and biomass from food and bioenergy crops, which are often polyploid and therefore difficult to manipulate genetically. The small size and easy growth requirements of Brachypodium allow for high through put genetic screens and gene discovery.

Acknowledgments We would like to thank Thomas Ream for critically reading the manuscript and Doobie Schmooney for many helpful insights. This work was funded by the US Department of Energy Great Lakes Bioenergy Research Center (http://www.great lakesbioenergy.org/).

\section{References}

1. Kobayashi Y, Weigel D (2007) Move on up, it's time for changemobile signals controlling photoperiod-dependent flowering. Genes Dev 21(19):2371-2384

2. Turck F, Fornara F, Coupland G (2008) Regulation and identity of florigen: FLOWERING LOCUS T moves center stage. Annu Rev Plant Biol 59:573-594

3. Kim D-H, Doyle MR, Sung S, Amasino RM (2009) Vernalization: winter and the timing of flowering in plants. Annu Rev Cell Dev Biol V 25:277-299

4. Greenup A et al (2009) The molecular biology of seasonal flowering-responses in Arabidopsis and the cereals. Ann Bot (Lond) 103(8):1165-1172

5. Loukoianov A et al (2005) Regulation of VRN-1 vernalization genes in normal and transgenic polyploid wheat. Plant Physiol 138(4):2364-2373

6. Yan L et al (2004) The wheat VRN2 gene is a flowering repressor down-regulated by vernalization. Science 303(5664):1640-1644

7. Yan L et al (2006) The wheat and barley vernalization gene VRN3 is an orthologue of FT. Proc Natl Acad Sci U S A 103(51):1958119586

8. Kojima S et al (2002) Hd3a, a rice ortholog of the Arabidopsis FT gene, promotes transition to flowering downstream of Hd1 under short-day conditions. Plant Cell Physiol 43(10):1096-1105

9. Opanowicz M et al (2008) Brachypodium distachyon: making hay with a wild grass. Trends Plant Sci 13(4):172-177

10. Garvin D, Gu Y, Hasterok R, Hazen S, Jenkins G, Mockler T, Mur LJ, Vogel JP (2008) Development of genetic and genomic research resources for Brachypodium distachyon, a new model for grass crop research. Crop Sci 48(S1):S69-S84

11. Draper J et al (2001) Brachypodium distachyon. A new model system for functional genomics in grasses. Plant Physiol 127(4):1539-1555

12. Foote TN et al (2004) Construction and analysis of a BAC library in the grass Brachypodium sylvaticum: its use as a tool to bridge the gap between rice and wheat in elucidating gene content. Funct Integr Genomics 4(1):26-33

13. Hasterok R et al (2006) Alignment of the genomes of Brachypodium distachyon and temperate cereals and grasses using bacterial artificial chromosome landing with fluorescence in situ hybridization. Genetics 173(1):349-362

14. Ozdemir BS et al (2008) Brachypodium genomics. Int J Plant Genomics 2008:536104

15. Olsen P, Lenk I, Jensen CS, Petersen K, Andersen CH, Didion T, Nielsen KK (2006) Analysis of two heterologous flowering genes in Brachypodium distachyon demonstrates its potential as a grass model plant. Plant Sci 170(5):1020-1025

16. Christiansen P, Andersen CH, Didion T, Folling M, Nielsen KK (2005) A rapid and efficient transformation protocol for the grass Brachypodium distachyon. Plant Cell Reports 23:751-758

17. Jensen CS, Salchert K, Nielsen KK (2001) A TERMINAL FLOWER1-like gene from perennial ryegrass involved in floral transition and axillary meristem identity. Plant Physiol 125 (3):1517-1528

18. Ohshima S, Murata M, Sakamoto W, Ogura Y, Motoyoshi F (1997) Cloning and molecular analysis of the Arabidopsis gene Terminal Flower 1. Mol Gen Genet 254(2):186-194

19. Shannon S, Meeks-Wagner R (1991) A mutation in the Arabidopsis TFL1 gene affects inflorescence meristem development. Plant Cell Physiol 3:877-892

20. Filiz E, Ozdemir BS, Tuna M, Budak H (2009) Diploid Brachypodium distachyon of Turkey: molecular and morphological analysis. In: Yamada T, Spangenberg G (eds) Molecular breeding of forage and turf. Springer, New York, pp 83-89 
21. Schmitz RJ et al (2005) FRIGIDA-ESSENTIAL 1 interacts genetically with FRIGIDA and FRIGIDA-LIKE 1 to promote the winter-annual habit of Arabidopsis thaliana. Development 132 (24):5471-5478

22. Hong SY et al (2008) Exploring valid reference genes for gene expression studies in Brachypodium distachyon by real-time PCR. BMC Plant Biol 8:112

23. Lang A (1965) Physiology of flower initiation. In: Ruhland W (ed) Encyclopedia of plant physiology. Springer, Berlin, pp 1371-1536

24. Purves WK, Sadava D, Orians GH (2004) Reproduction in flowering plants. In: Sadava D, Craig Heller H, Orians GH, Purves WK, Hillis DM (eds) Life: the science of biology: volume III: plants and animals. Macmillan, Basingstoke

25. Evans LT (1975) The physiological basis of crop yield. In: Evans LT (ed) Crop physiology: some case histories. Cambridge University Press, Cambridge

26. Trevaskis B et al (2006) HvVRN2 responds to daylength, whereas HvVRN1 is regulated by vernalization and developmental status. Plant Physiol 140(4):1397-1405

27. Dubcovsky J et al (2006) Effect of photoperiod on the regulation of wheat vernalization genes VRN1 and VRN2. Plant Mol Biol 60 (4):469-480
28. Distelfeld A, Li C, Dubcovsky J (2009) Regulation of flowering in temperate cereals. Curr Opin Plant Biol 12(2):178-184

29. Trevaskis B et al (2007) The molecular basis of vernalizationinduced flowering in cereals. Trends Plant Sci 12(8):352-357

30. Purugganan MD et al (1995) Molecular evolution of flower development: diversification of the plant MADS-box regulatory gene family. Genetics 140(1):345-356

31. Nam J et al (2003) Antiquity and evolution of the MADS-box gene family controlling flower development in plants. Mol Biol Evol 20(9):1435-1447

32. Vogel JP, Metin T, Budak H, Huo N, Gu YQ, Steinwand MA (2009) Development of SSR markers and analysis of diversity in Turkish populations of Brachypodium distachyon. BMC Plant Biol 9(88):1-11

33. Distelfeld A et al (2009) Genetic and molecular characterization of the VRN2 loci in tetraploid wheat. Plant Physiol 149(1):245-257

34. Vogel J, Garvin DF, Leong O, Hayden D (2006) Agrobacteriummediated transformation and inbred line development in the model grass Brachypodium distachyon. Plant Cell Tiss Org Cult 85:199-211

35. Vogel J, Hill T (2008) High-efficiency Agrobacterium-mediated transformation of Brachypodium distachyon inbred line Bd21-3. Plant Cell Rep 27(3):471-478 